\title{
Previous Malignancy as a Risk Factor for the Second Solid Cancer in a Cohort of Nuclear Workers
}

\author{
Mikhail Osipov $^{1 *}$, Mikhail Sokolnikov ${ }^{2}$ \\ ${ }^{1}$ Southern Urals Biophysics Institute, Ozyorskoye Shosse 19, Ozyorsk 456780, Russian Federation.
}

Received 14 August 2020; Revised 13 November 2020; Accepted 21 January 2021; Published 01 March 2021

\begin{abstract}
This paper describes the results of epidemiological analysis of a cohort of nuclear workers hired at the main facilities of "Mayak" Production Association located in the city of Ozyorsk in Southern Urals of the Russian Federation. Previous malignancy as a risk factor for second cancer in a cohort of 22,373 workers occupationally exposed to ionizing radiation has been analyzed in a retrospective study with more than 60 years of follow-up. Information on main radiation and nonradiation risk factors (attained age, sex, tobacco smoking), as well as the dose of exposure to occupational ionizing radiation has been obtained for the analysis using the data from cancer register as well as other main population registries created in Epidemiological Laboratory of Southern Urals Biophysics Institute. Poisson's regression realized in the "Amfit" module of "Epicure" statistical package has been applied for risk analysis. Excess relative risk per $1 \mathrm{~Gy}$ of absorbed dose of external gamma radiation and internal alpha radiation has been calculated using linear model. Among the 2,471 cancer cases accumulated in the study cohort to the end of follow-up $6.4 \%$ of second cancer cases have been diagnosed among workers occupationally exposed to protracted external gamma- and internal alpha radiation. The relative risk of second cancer (except for non-melanoma skin cancer) among nuclear workers with previously diagnosed cancer was about 4 times higher after a decade compared with those cancer patients who had single cancer only. The results showed that previous malignancy along with main non-radiation factors is statistically significant carcinogenic risk factor among nuclear workers exposed to protracted occupational radiation.
\end{abstract}

Keywords: Malignant Neoplasms; Second Cancer; Previous Malignancy; Subsequent Cancer; Occupational Exposure; Carcinogenic Risk.

\section{Introduction}

Malignant Neoplasms (MN) is one of the major problems in a global health care system today, as one of the most potentially life-threatening conditions. Each year in Russia about 190,000 people diagnosed with cancer become disabled ( $30 \%$ of them with a severe disability), and the proportion of new cancer cases among the patients with a severe disability is more than $41 \%$ [1]. Spending on medical insurance in regard to the high cost of treatment and rehabilitation of cancer patients requires taking measures primarily aimed at cancer prevention. On the other hand, growing healthcare quality resulted in the increase in survival of cancer patients. According to the National Cancer Institute of the United States, the 5-year survival for all malignancies in the last decade increased from 50 to $66 \%$ in adults and the 10-year survival rate has reached $59 \%$. At the same time, those survival advances have been offset by the long-term effects of cancer and its treatment, particularly second MN. The probability of subsequent malignant tumors development among the patients already diagnosed with cancer is 1.14 times higher than in the general population [2], particularly among those patients who had received anti-cancer therapy [3].

\footnotetext{
* Corresponding author: osipov@subi.su

$>$ This is an open access article under the CC-BY license (https://creativecommons.org/licenses/by/4.0/).

(C) Authors retain all copyrights. 
The main goal of cancer prevention programs worldwide is to control potential risk factors, including Ionizing Radiation (IR) and MN itself as probable carcinogens. Epidemiological analysis of cancer survivors remains the unique approach in assessing their role in carcinogenesis and provides the scientific basis for cancer prevention measures. Epidemiological studies are highly important in populations occupationally exposed to IR, such as nuclear workers, where the risk of cancer is higher than in general population [4]. However, as usual practice, only the first cases of cancer are used for the epidemiological analysis (i.e. date of the first diagnosis of cancer is the date of end of the follow-up) [5], despite the fact that the second cancer may occur after the first malignancy. The purpose of the study was to evaluate the influence of previous cancer on developing second primary malignancy in a cohort of nuclear workers exposed to IR as an additional risk factor.

\section{Materials and Methods}

\subsection{Materials}

A retrospective cohort study has been conducted using the Mayak Worker Cohort (MWC) data - the cohort of nuclear workers of the "Mayak" Production Association (MPA), located in the Southern Urals of Russia $10 \mathrm{~km}$ far from the city of Ozyorsk. The cohort includes 22,373 individuals over the age of 18 hired on reactor, radiochemical or plutonium production facility of MPA in the period from 1948 to 1972.

The follow-up period started from the date of first employment of a worker to a main MPA facility and ended on the date of the last diagnosis of malignancy, or the date of death, or migration date (i.e. leaving the place of residence without any further information), or the date of the end of the follow-up period (December, 31, 2016), whichever the earliest. The procedure for collection and verification of data, stating the vital status as well as collecting the medical information including tobacco smoking for the workers was described in details in Koshurnikova et al. (1999) study [6].

The main source of medical information was local Cancer Register, created in the epidemiology laboratory of Southern Urals Biophysics Institute. Cancer Register is the population-based registry which contains information on 13,899 cancer cases diagnosed through the period from 1948 to 2016 among Ozyorsk population of all ages. The Cancer Register contains information on the date of cancer detection, diagnosis of MN, stage of malignant process and the histological data, as well as additional information. The cases of the second primary MN have been determined according to Warren Gates (1932) criteria for a diagnosis of Multiple Primary Malignancies (MPM):

(a) Each tumor should present a definite picture of malignancy;

(b) Each tumor should be histologically distinct;

(c) The possibility that one MN is metastasis of the other must be excluded.

The second source of information for vital status assessment was the "Child Register" contained information on the vital status and the cause of death for 92,751 persons who came to Ozyorsk at the age under 15 or were born in Ozyorsk in the period through 1946 to 1984 . An additional information on the vital status of the cohort members has been obtained from the "CT Register" which contains information on 26,626 computed tomography examinations during the period from 1993 to 2020. The detailed information on this data source can be found in the Fomin and Osipov (2019) study [7]. The main dosimetric characteristics of the Mayak personnel using the Mayak worker dosimetry system (MWDS) "Dose2008" were given in Romanov et al. (2002) work [8]. The cumulative individual absorbed dose of external gamma radiation $(\gamma)$ and internal alpha radiation $(\alpha)$ from MWDS has been used for the analysis. The flow chart of the study describing main sources of information and the process of data binding is presented in Figure 1.

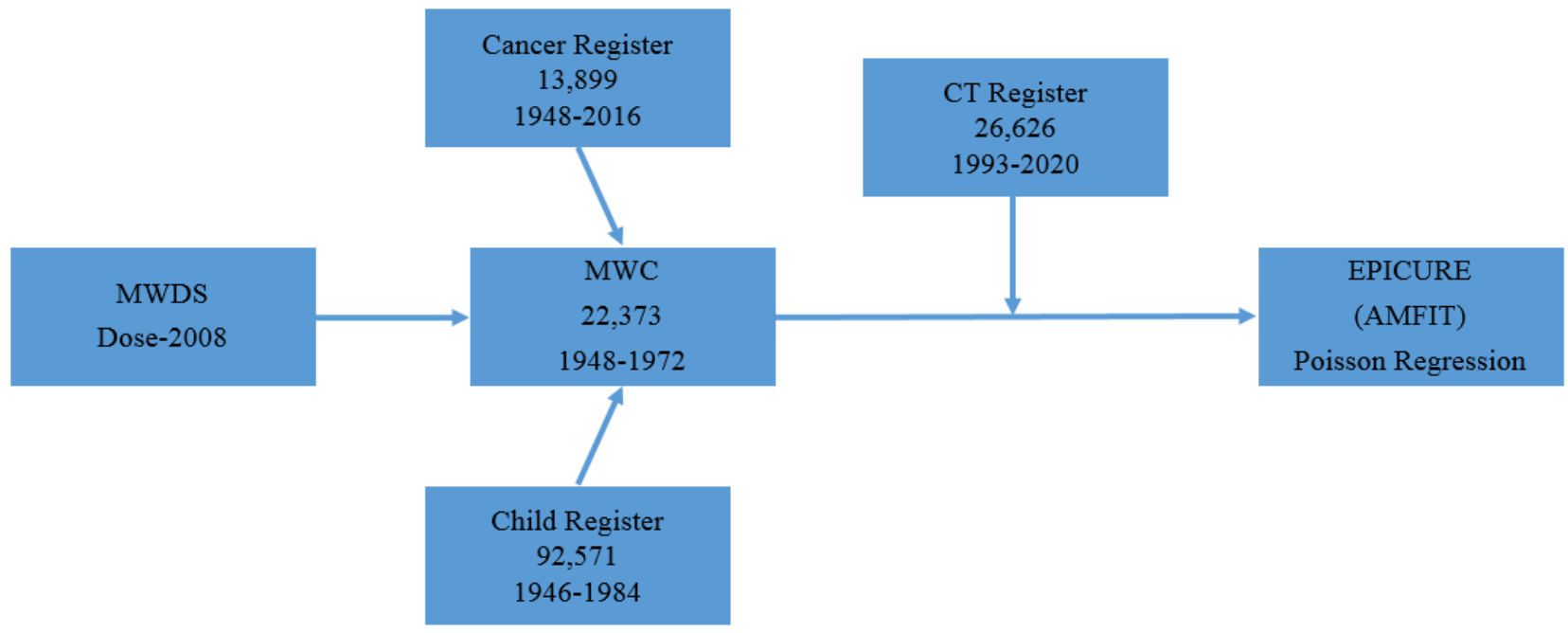

Figure 1. The flow chart of the study 


\subsection{Statistical Modelling}

Piece-wise Poisson's exponential regression implemented in "Amfit" module of "Epicure" statistical package [9] has been used for statistical modelling. Product additive excess model has been tested for linearity using maximum likelihood method. The general model of cancer incidence $(\lambda)$ with "background" $\left(\lambda_{0}\right)$ and "excess" (ERR) components is given in Equation 1:

$\lambda=\lambda_{0} \times(1+\mathrm{ERR})$

Multiplicative background risk model depending on main non-radiation risk factors such as sex $(\mathrm{m}=\mathrm{male}, \mathrm{f}=$ female), age attained to the date of the first malignancy (age), birth cohort which describes the changes in cancer incidence in the cohort over decades (bcoh) and tobacco smoking (smk) was created with the assumption that previous cancer increases the risk of second primary cancer (Equation 2):

$\lambda_{0}=\exp \left(m+f+\ln \left[\frac{\text { age }}{60}\right] \times \operatorname{sex}+\ln \left[\frac{\text { age }}{60}\right]^{2} \times \operatorname{sex}+s m k \times s e x+b c o h+p c\right)$

The data on tobacco smoking has been categorized (variable "smk"). This parameter was defined as " 0 " for never smoked, "1" for those who had ever been a smoker and " 2 " for those who had no information on smoking. The variable describing birth cohort ("bcoh") has been defined as "0" for workers hired on MPA before 1953 (the early period of MPA operation), "1" for those hired between 1953 and 1957, " 2 " for workers hired between 1957 and 1963, and " 3 " for workers hired between 1963 and 1972 that reflected changes in cancer incidence due to calendar time. The data on previous malignancy has been described with "pc" variable which indicates the presence of previous cancer diagnosed before the date of last solid cancer diagnosis with 2, 5 and 10-year lag.

\section{Results}

\subsection{Vital Status}

The study cohort included 22,373 nuclear workers (16,686 males and 5,687 females) hired at the main facilities of MPA (reactor, radiochemical and plutonium production) in the period since 1948 to $1972.58 .4 \%$ of the cohort members were Ozyorsk residents until the end of the follow-up. For $41.2 \%$ of the cohort members the date of migration (i.e. leaving the place of residence in Ozyorsk) was the date of end of the follow-up. For a small percentage ( $0.3 \%)$ of workers the last known date was the date of last operation at MPA. Detailed information on the vital status of the workers by sex is presented in Table 1 .

Table 1. Vital status of workers in MWC by sex as of December, 31, 2016

\begin{tabular}{cccc}
\hline Vital status & Male (\%) & Female (\%) & Both sex (\%) \\
\hline Alive & 36.6 & 48.3 & 39.6 \\
Dead & 58.0 & 46.9 & 55.2 \\
Lost & 5.4 & 4.8 & 5.2 \\
Total & 100.0 & 100.0 & 100.0 \\
\hline
\end{tabular}

More than $50 \%$ of the cohort members had died to the end of the follow-up, and $5.2 \%$ of persons were lost to the follow-up. In present study $94.8 \%$ of the cohort members with known vital status have been used for the analysis.

\subsection{Cancer Incidence in the Study Cohort}

2,471 cases of MN had been accumulated in the study cohort to the end of the follow-up. The distribution of the cohort members by the total number of cancer cases per person (including non-melanoma skin cancer, NMSC) as of December, 31, 2016 in the study cohort by sex is shown in Table 2.

Table 2. The number of MN in the study cohort

\begin{tabular}{cccc}
\hline Number of MN & Male (\%) & Female (\%) & Both sex (\%) \\
\hline 1 & $1,671(93.6)$ & $642(93.7)$ & $2,313(93.6)$ \\
2 & $104(5.8)$ & $42(6.1)$ & $146(5.9)$ \\
3 & $9(0.5)$ & $0(0.0)$ & $9(0.4)$ \\
4 & $1(0.1)$ & $1(0.1)$ & $2(0.08)$ \\
5 & $1(0.1)$ & $0(0.0)$ & $1(0.04)$ \\
Total & 1,786 & 685 & 2,471 \\
\hline
\end{tabular}


Male to female ratio among workers diagnosed with cancer was 2.6 (while male to female ratio in the whole cohort was 2.9). Among all 2,471 cancer patients in the study cohort $93.6 \%$ of patients had a single MN diagnosed during the follow-up period, with the proportion of multiple primary solid cancers of $6.4 \%$.

Among 158 cancer patients with the second malignancy 146 persons (92.4\%) had two malignant neoplasms diagnosed in the period from 1948 to 2016, and 7.6\% of workers had several malignant tumors (up to 5 MN per person), mostly due to non-melanoma skin cancer $(27 \%)$. Since basal cell carcinomas (i.e. NMSC) have a tendency to recur, causing multiple registration of one malignancy, a common practice is to exclude NMSC from analysis. Except for NMSC, the total number of cancer cases in the study cohort to the end of the follow-up was 116 with $94 \%$ of solid tumours. The distribution of the first and second MN except for NMSC by age attained is shown on Figure 2.

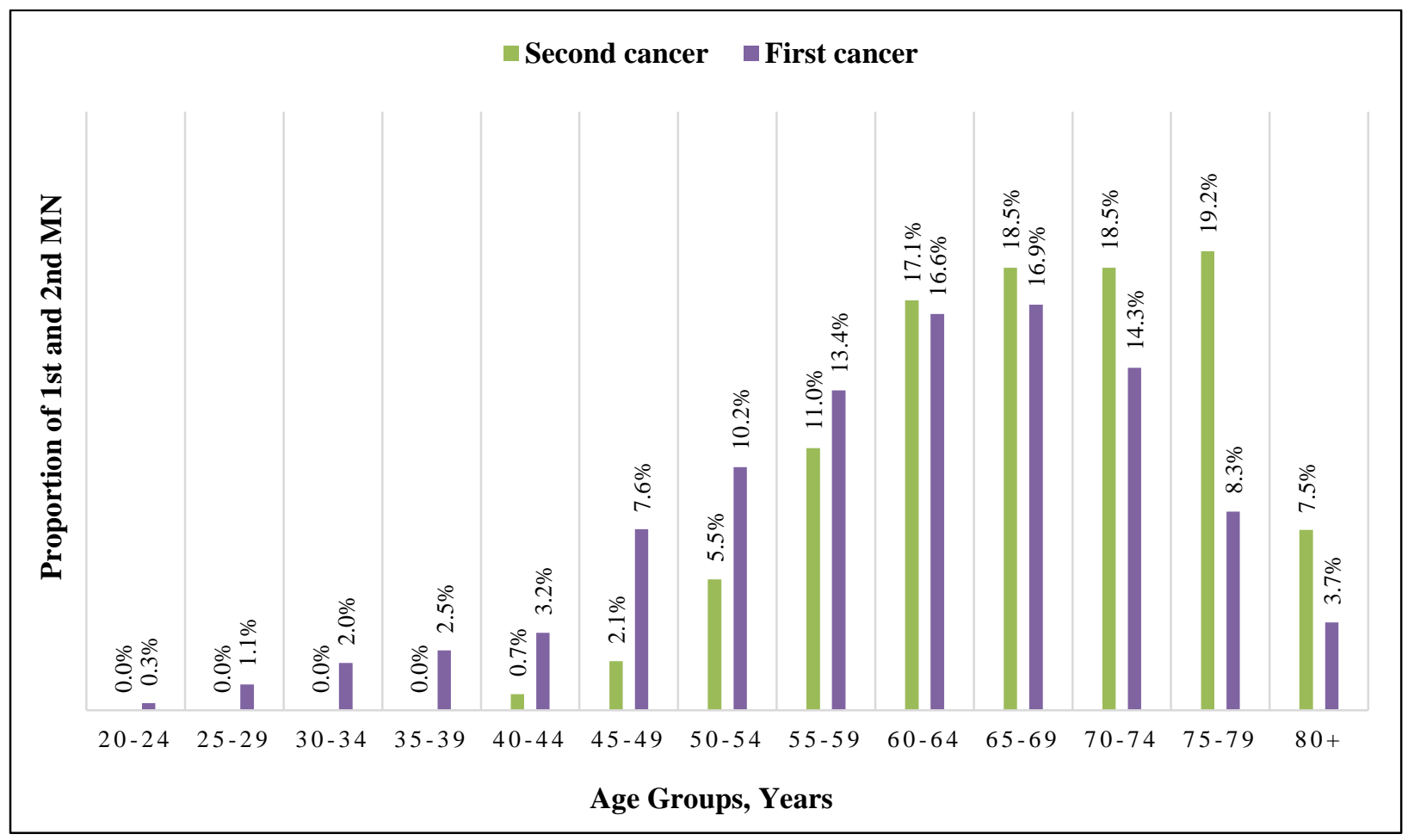

Figure 2. The distribution of $1^{\text {st }}$ and $2^{\text {nd }} \mathrm{MN}$ by age attained (excluding NMSC)

The mean age attained to the date of the $1^{\text {st }}$ malignancy was 57.1 years $(95 \%$ CI: $54.9 ; 59.3)$, with median of 59 years. The first cancer cases in the cohort were diagnosed at the age after 21 years, while the second cases of solid cancer started to appear after the age of 40 years, with mean age attained to the date of second MN of 67.5 years (95\% CI: 65.9; 69.1). Mean period between the $1^{\text {st }}$ and $2^{\text {nd }}$ malignancy was 10.4 years ( $\max 45$ years) with $70.8 \%$ of second solid cancer cases diagnosed within 5 years from $1^{\text {st }}$ cancer. The structure of the $1^{\text {st }}$ and the $2^{\text {nd }}$ malignancy by ICD-9 classification and $2^{\text {nd }}$ to $1^{\text {st }}$ ratio is shown in Table 3.

Table 3. Cancer incidence in the study cohort by ICD-9 groups

\begin{tabular}{ccccc}
\hline ICD-9 code & Localization & $\mathbf{1}^{\text {st }}$ malignancy, $\boldsymbol{\%}$ & $\mathbf{2}^{\text {nd }}$ malignancy, $\%$ & $\mathbf{2}^{\text {nd }} / \mathbf{1}^{\text {st }}$ ratio \\
\hline $140-149$ & Lips, oral cavity and pharynx & 11.2 & 5.2 & 0.46 \\
$150-159$ & Abdominal & 21.6 & 31.0 & 1.44 \\
$160-165$ & Respiratory & 25.0 & 24.1 & 0.97 \\
162 & Lung & 21.6 & 22.4 & 1.04 \\
$170-175^{*}$ & Bone, skin, connecting tissue and breast & 17.2 & 9.5 & 0.55 \\
174 & Breast & 11.2 & 6.0 & 0.54 \\
$180-189$ & Urogenital & 17.2 & 24.1 & 1.40 \\
185 & Prostate & 2.6 & 7.8 & 3.0 \\
$190-199$ & CNS, thyroid and other & 4.3 & 0 & 0.0 \\
$200-203$ & Lymphoma & 2.6 & 0.9 & 0.33 \\
$204-208$ & Leukemia & 0.9 & 5.2 & 6.0 \\
\hline
\end{tabular}

*except for NMSC 
The most common cancer localization among the $2^{\text {nd }}$ solid malignancies was lung cancer $(22.4 \%)$. Stomach and prostate cancer (equally $7.8 \%$ ), breast cancer (6\%), colorectal cancer (13.8\%), and kidney cancer $(7.6 \%)$ were the rest of sites which exceeded $5 \%$ of all second cancer cases accumulated in the study cohort to the end of follow-up. The " $2^{\text {nd }}$ to $1^{\text {st" }}$ ratio shows that some cancers more often appear as a second malignancy. The most common sites for the $2^{\text {nd }}$ cancer are leukemia, prostate cancer and gastrointestinal cancers.

\subsection{Risk Analysis}

Results of the relative risk (RR) analysis of influence of a previous malignancy with 0,2 and 5 years between previous and second solid cancer, adjusted for sex, age attained and smoking in the model of excess relative risk (ERR) using cumulative external dose of gamma radiation $(\gamma)$ and cumulative dose of internal alpha radiation $(\alpha)$ are shown in Table 4.

Table 4. RR estimates depending on lag period and ERR/Gy for external gamma radiation (ERR $\gamma$ ) and internal alpha radiation (ERR $\alpha$ )

\begin{tabular}{cccc}
\hline Lag period & $\mathbf{R R}(\mathbf{9 5 \%} \mathbf{C I})$ & $\mathbf{E R R}_{\boldsymbol{u}}(\mathbf{9 5 \%} \mathbf{C I})$ & $\mathbf{E R R}_{\gamma}(\mathbf{9 5 \%} \mathbf{C I})$ \\
\hline Overall RR & $3.9(3.6 ; 4.5)$ & $0.08(-0.05 ; 0.18)$ & $0.08(-0.03 ; 0.19)$ \\
2 years & $4.2(3.6 ; 4.7)$ & $0.09(-0.03 ; 0.17)$ & $0.09(-0.02 ; 0.2)$ \\
5 years & $4.6(3.9 ; 5.3)$ & $0.09(-0.002 ; 0.2)$ & $0.09(0.0004 ; 0.18)$ \\
10 years & $6.9(4.8 ; 9.7)$ & $0.1(-0.02 ; 0.21)$ & $0.1(0.003 ; 0.19)$ \\
\hline
\end{tabular}

As shown in Table 4, overall risk ratio of the second solid cancer diagnosed years after the previous malignancy made 3.9 compared to the workers with a single MN. The results indicate that background incidence in the study cohort in the presence of previous cancer will be 4 times higher compared to those who have a single cancer diagnosed during the follow-up. Along with that, for male smokers who had previous cancer the baseline cancer incidence was 1.4 (95\% CI: $1.19 ; 1.66)$ in comparison to those who have never been smokers, along with no significant increase for female smokers $(1.3,95 \%$ CI: $0.75 ; 2.2)$. RR increased with the time between previous and the second solid cancer with increasing ERR per $1 \mathrm{~Gy}$ of absorbed dose of external gamma radiation and internal alpha radiation, which corresponds to the results of Osipov and Sokolnikov (2014) [10]. The impact of other non-radiation factors such as birth cohort $(p<0.0005)$ has been also found significant.

\section{Discussion}

These findings indicate a significant correlation between a previous malignancy and the second solid cancer development in a cohort of nuclear workers exposed to protracted external gamma- and internal alpha occupational radiation. Based on this result, there is a possibility that, being previously diagnosed with cancer, nuclear workers have an increased risk of development of the second malignancy decades after. According to that, one may come to a conclusion that a previous malignancy may play an etiological role in the development of the second cancer. However, it is unclear whether this is a consequence of (1) direct carcinogenic effect of a previous malignant tumor, or (2) result of cancer treatment process, related to diagnostic radiation exposure, radiotherapy and chemotherapy (CRT), as known possible carcinogens [11].

Among the mentioned above, CRT seems to confer higher risks for solid cancer after a primary cancer, though the risks appear to be lower in patients exposed to radiotherapy at older ages. Thus, we cannot reject completely either the first or the second assumption. Moreover, mutual influence of these conditions is likely. Besides, in this study we did not analyze the effects of separate cancer sites (for instance, the lung cancer, which is probably affected by occupational exposure to internal alpha-radiation; leukemia, and sex-depended MN - prostate and breast cancer) due to small number of cases. Thus, we cannot assert whether each type of malignancy plays equal role in the excess number of cases among persons having second MN. However, we used " $2^{\text {nd }}$ to $1^{\text {stc }}$ malignancy ratio to show that leukemia (6.0) and prostate cancer (3.0) have more frequently appeared after the first cancer compared to the rest of the second MN (for instance, lung cancer was relatively rare consequence of previous malignancy). Increased " $2^{\text {nd }}$ to $1^{\text {stc }}$ ratio for leukemia as a second cancer in this study supports the notion that leukemia can also be promoted by conditions related to cancer treatment process [12]. Regarding prostate cancer, this assumption is consistent with the latest studies on the risk assessment of prostate cancer after radiation therapy [13]. 51\% of the first cancers followed by prostate cancer during the decade of follow-up study [14] that might suggest that this localization is more sensitive to the influence of factors associated with the presence of a previous cancer, including diagnostic and therapeutic exposure, what is the point of interest for further research.

Although radiotherapy probably plays essential role in development of subsequent malignancy, the data on the patient's dose from radiotherapy were not available in this study. This can serve as a source of uncertainty in radiation risk assessment, since the dose received by a patient during radiotherapy can be quite high compared to diagnostic or 
occupational radiation. Moreover, the dose from radiotherapy can vary greatly depending on localization of a malignant process. It is undoubtedly, that in future research work on the assessment of radiation risk, it is necessary to take into account the influence of all factors in order for the estimates obtained to be valid.

According to (Murphy, 2018) $1 / 4$ of cancer patients in the U.S. population aged after 65 years and more than $10 \%$ of younger adults newly diagnosed with cancer have a previous cancer; the prevalence of prior cancer ranged from $3.5 \%$ to $36.9 \%$ differed by incident cancer type [15]. In a recent study of Japanese cancer register (Sato, 2020) in a cohort of 275,895 patients [16] there was a higher prevalence of the second cancers (7.9\%), compared to $6.4 \%$ in our study cohort, that may indicate population differences other than age and sex, apparently related to different diagnostics and treatment, and other factors such as survival period.

Since mean period between the $1^{\text {st }}$ and the $2^{\text {nd }}$ malignant tumors in our study reached 10 years (with maximum of 45 years), there is a reason to treat the second malignant tumors as a multiple primary malignancy (MPM) with independent growth. However, taking into account the long latency period for several types of tumors, it cannot be ruled out whether those observed second cancers are synchronous (up to 6 months between the onsets) or metachronous (more than 6 months after the first MN), since the exact date of the onset of the subsequent tumor cannot be established by the existing diagnostic methods.

In addition to the mentioned above, in this study the reverse causation (i.e. confounding by indication) cannot be neglected, since the cancer patients were observed more carefully compared to non-cancer patients (this phenomenon has also been described by Boice (2015) [17], as well as in our previous work focused on low dose from diagnostic exposure among nuclear workers [18]. Nevertheless, in case of repeated diagnostic exposure of cancer patients it makes sense to use algorithms for reducing the radiation dose, especially in patients undergoing anticancer treatment [19]. Currently, such protocols are already in use in our healthcare system. Perhaps this is one of the reasons for the lack of influence of the number of diagnostic procedures on occurrence of subsequent cancers, as it shown in Osipov et al. (2020) [20]. However, this issue requires further consideration because of relatively short follow-up period reported.

Finally, the cohort of MPA workers has its own features that determine the unique nature of the study. First, the state of health of the workers was better at the moment of employment at nuclear facilities of MPA [4]. On the other hand, occupational exposure to IR was an additional carcinogenic risk factor which can influence the prevalence of the second primary $\mathrm{MN}$ in the study cohort. Another peculiarity of this research is the age distribution with zero cases under 18 due to occupational reasons. These factors impose certain restrictions on comparing the results with other similar studies.

\section{Conclusion}

The results of the study showed that, along with known radiation risk modifiers in a cohort of nuclear professionals such as sex, attained age and tobacco smoking, the presence of a previous cancer is also a significant non-radiation risk factor that should be considered in epidemiological research study. Although the etiological role of previous cancer in the development of the second primary malignant neoplasm remains unclear, the inclusion of this factor into the model of background cancer incidence will make it possible to assess the radiation risk without limitations caused by using the first cancer cases only. As for the radiation safety of cancer patients undergoing medical diagnostic exposure to ionizing radiation such as CT and PET/CT, the attending physician and radiologist should be careful when prescribing such diagnostic procedures to prevent the possible risk of development of subsequent cancers. Radiologists and physicians should also integrate age, sex, smoking status, projected life expectancy when deciding upon diagnostic radiation procedures. Further evidence-based guidelines are needed for early detection and treatment of the second malignancies in the group of cancer patients occupationally exposed to ionizing radiation. Elaboration of additional radiation protection measures will help to reduce possible late carcinogenic effects of the first malignancy.

\section{Declarations}

\subsection{Author Contributions}

Conceptualization, M.O. and M.S.; methodology, M.O. and M.S.; formal analysis, M.O. and M.S.; writing-review and editing, M.O. and M.S. All authors have read and agreed to the published version of the manuscript.

\subsection{Funding}

The author(s) received no financial support for the research, authorship, and/or publication of this article.

\subsection{Acknowledgements}

The authors are grateful to Southern Urals Biophysics Institute (SUBI) International Department team. 


\subsection{Ethical Approval}

The manuscript does not contain experimental data on animals or humans; hence, ethical permission is not required.

\subsection{Data Availability Statement}

The data presented in this study are available in article.

\subsection{Conflict of Interest}

The authors declare that they have no known competing financial interests or personal relationships that could have appeared to influence the work reported in this paper.

\section{References}

[1] Caprin, A. D., Starinsky, V. V., \& Petrova, G. V. (2019). Malignant neoplasms in Russia in 2018 (morbidity and mortality) Moscow: MNIOI them. PA Herzen branch of the Federal State Budgetary Institution" National Medical Research Center of Radiology" of the Ministry of Health of Russia.

[2] Curtis R.E., Freedman D.M., Ron E., Ries L.A.G., Hacker D.G., Edwards B.K., Tucker M.A., Fraumeni J.F. Jr., eds. (2006). New Malignancies among Cancer Survivors: SEER Cancer Registries, 1973-2000. National Cancer Institute. NIH Publ. No. 05-5302. Bethesda, MD.

[3] Rees, J. R., Zens, M. S., Gui, J., Celaya, M. O., Riddle, B. L., \& Karagas, M. R. (2014). Non Melanoma Skin Cancer and Subsequent Cancer Risk. PLoS ONE, 9(6), e99674. doi:10.1371/journal.pone.0099674.

[4] Sokolnikov, M. E., Gilbert, E. S., Preston, D. L., Ron, E., Shilnikova, N. S., Khokhryakov, V. V., ... Koshurnikova, N. A. (2008) Lung, liver and bone cancer mortality in Mayak workers. International Journal of Cancer, 123(4), 905-911. doi:10.1002/ijc.23581

[5] Preston, D. L., Ron, E., Tokuoka, S., Funamoto, S., Nishi, N., Soda, M., ... Kodama, K. (2007). Solid Cancer Incidence in Atomic Bomb Survivors: 1958-1998. Radiation Research, 168(1), 1-64. doi:10.1667/rr0763.1.

[6] Koshurnikova, N. A., Shilnikova, N. S., Okatenko, P. V., Kreslov, V. V., Bolotnikova, M. G., Sokolnikov, M. E., ... Romanov, S. A. (1999). Characteristics of the Cohort of Workers at the Mayak Nuclear Complex. Radiation Research, 152(4), 352. doi: $10.2307 / 3580220$.

[7] Fomin, E. P., \& Osipov, M. V. (2019). Pooled Database of Ozyorsk Population Exposed to Computed Tomography. Russian Electronic Journal of Radiology, 9(2), 234-239. doi:10.21569/2222-7415-2019-9-2-234-239.

[8] Romanov, S., Vasilenko, E., Khokhryakov, V., \& Jacob, P. (2002). Studies on the Mayak nuclear workers: dosimetry. Radiation and Environmental Biophysics, 41(1), 23-28. doi:10.1007/s00411-001-0134-3.

[9] D. L. Preston, J. H. Lubin, D. A. Pierce, and M. E. McConney. (1993). Epicure: User's Guide. HiroSoft International Corporation, Seattle, WA, 1993.

[10] Osipov M.V., Sokolnikov M.E. (2014). Digestive Tract Cancer Risk Assessment among "Mayak" PA Workers. Questions of Radiation Safety. 1: 76-83.

[11] VanderWalde, A. M., \& Hurria, A. (2011). Second Malignancies among Elderly Survivors of Cancer. The Oncologist, 16(11), 1572-1581. doi:10.1634/theoncologist.2011-0214.

[12] Zhang, B., Zhang, X., Li, M., Kong, L., Deng, X., \& Yu, J. (2016). How breast cancer chemotherapy increases the risk of leukemia: Thoughts about a case of diffuse large B-cell lymphoma and leukemia after breast cancer chemotherapy. Cancer Biology \& Therapy, 17(2), 125-128. doi:10.1080/15384047.2016.1139233.

[13] Boehle, A., Katic, K., König, I. R., Robrahn-Nitschke, I., \& Brandenburg, B. (2020). Comparison of outcome endpoints in intermediate- and high-risk prostate cancer after combined-modality radiotherapy. Brachytherapy, 19(1), $24-32$. doi:10.1016/j.brachy.2019.09.001.

[14] Dong, C., \& Hemminki, K. (2001). Second primary neoplasms in 633,964 cancer patients in Sweden, 1958-1996. International Journal of Cancer, 93(2), 155-161. doi:10.1002/ijc.1317.

[15] Murphy, C. C., Gerber, D. E., \& Pruitt, S. L. (2018). Prevalence of Prior Cancer Among Persons Newly Diagnosed With Cancer: An Initial Report From the Surveillance, Epidemiology, and End Results Program. JAMA Oncology, 4(6), 832-836. doi:10.1001/jamaoncol.2017.3605.

[16] Sato, A., Matsubayashi, K., Morishima, T., Nakata, K., Kawakami, K., \& Miyashiro, I. (2020). Increasing Trends in the Prevalence of Prior Cancer in Newly Diagnosed Lung, Stomach, Colorectal, Breast, and Uterine Cancer Patients: A PopulationBased Study. doi:10.21203/rs.3.rs-41046/v1. 
[17] Boice, J. D. (2015). Radiation epidemiology and recent paediatric computed tomography studies. Annals of the ICRP, 44(1_suppl), 236-248. doi:10.1177/0146645315575877.

[18] Osipov, M. V., \& Sokolnikov, M. E. (2015). Problems of assessment of carcinogenic risk of medical exposure in a cohort of nuclear personnel. Meditsinskaya radiologiya i radiatsionnaya bezopasnost, 60(6), 60-66.

[19] Osipov, M. V., Lebedev, N. I., \& Fomin, E. P. (2015). Radiation Safety of Patients: Reducing the Radiation Dose in Abdominal Multislice Computed Tomography. Russian Electronic Journal of Radiology. 2015; 5 (2): 47-51 (in Russian).

[20] Osipov, M., Vazhenin, A., Kuznetsova, A., Aksenova, I., Vazhenina, D., \& Sokolnikov, M. (2020). PET-CT and Occupational Exposure in Oncological Patients. SciMedicine Journal, 2(2), 63-69. doi:10.28991/scimedj-2020-0202-3. 